\title{
Self-injurious behaviour: limbic dysregulation and stress effects in an animal model
}

\author{
A. M. Muehlmann, ${ }^{1,2}$ S. D. Kies,' C. A. Turner, ${ }^{3}$ S. Wolfman,' M. H. Lewis ${ }^{2}$ \& D. P. Devine' \\ I Behavioral and Cognitive Neuroscience Program, Department of Psychology, University of Florida, Gainesville, Florida, USA \\ 2 Department of Psychiatry, University of Florida, Gainesville, Florida, USA \\ 3 Molecular and Behavioral Neuroscience Institute, University of Michigan, Ann Arbor, Michigan, USA
}

\begin{abstract}
Background Self-injurious behaviour (SIB) is prevalent in neurodevelopmental disorders, but its expression is highly variable within, and between diagnostic categories. This raises questions about the factors that contribute to aetiology and expression of SIB. Expression of SIB is generally described in relation to social reinforcement. However, variables that predispose vulnerability have not been as clearly characterised. This study reports the aetiology and expression of self-injury in an animal model of pemoline-induced SIB. It describes changes in gross neuronal activity in selected brain regions after chronic treatment with pemoline, and it describes the impact that a history of social defeat stress has on the subsequent expression of SIB during pemoline treatment.

Methods Experiment I - Male Long-Evans rats were injected on each of five consecutive days with pemoline or vehicle, and the expression of SIB was evaluated using a rating scale. The brains were harvested on the morning of the sixth day, and were assayed for expression of cytochrome oxidase, an index of sustained neuronal metabolic activity. Experiment 2 - Male Long-Evans rats were exposed to a regimen of 12 daily sessions of social defeat stress or 12 daily sessions of handling (i.e. controls). Starting on the day after completion of the social defeat or handling regimen, each rat was given five

Correspondence: Dr Darragh P. Devine, Behavioral and Cognitive Neuroscience Program, Department of Psychology, University of Florida, PO Box II2250, Center Drive, Gainesville, FL 326I I2250, USA (e-mail: dpdevine@ufl.edu).
\end{abstract}

daily injections of pemoline. The durations of selfinjurious oral contact and other stereotyped behaviours were monitored, and the areas of tissue injury were quantified.

Results Experiment I - Neuronal metabolic activity was significantly lower in a variety of limbic and limbic-associated brain structures in the pemolinetreated rats, when compared with activity in the same regions of vehicle-treated controls. In addition, neuronal activity was low in the caudateputamen, and in subfields of the hypothalamus, but did not differ between groups for a variety of other brain regions, including nucleus accumbens, substantia nigra, ventral tegmentum, thalamus, amygdala, and cortical regions. Experiment 2 - All the pemoline-treated rats exhibited SIB, and whereas the social defeat regimen did not alter the total amount of self-injurious oral contact or other stereotyped behaviours, it significantly increased the severity of tissue injury.

Conclusions A broad sampling of regional metabolic activity indicates that the pemoline regimen produces enduring changes that are localised to specific limbic, hypothalamic and striatal structures. The potential role of limbic function in aetiology of SIB is further supported by the finding that pemolineinduced self-injury is exacerbated by prior exposure to social defeat stress. Overall, the results suggest brain targets that should be investigated further, and increase our understanding of the putative role that stress plays in the pathophysiology of SIB.

Keywords animal model, Lesch-Nyhan syndrome, pemoline, self-injury, social defeat, stress 
A. M. Muehlmann et al. - Stress and SIB in the pemoline model

\section{Introduction}

Self-injurious behaviour (SIB) is a debilitating characteristic that is exhibited by individuals with a broad variety of neurodevelopmental disorders (Rojahn \& Esbensen 2002). It is seen in virtually all people with Lesch-Nyhan syndrome (Lesch \& Nyhan 1964; Schretlen et al. 2005), most people with Prader-Willi syndrome (Symons et al. 1999) and approximately one-third of children with autism (Matson et al. I996). Interestingly, it is not a definitive trait of most diagnostic categories. Rather, expression is heterogeneous within most of these groups, but shared across diagnoses. This suggests that characteristics that are symptomatic of multiple forms of intellectual disabilities may tend to be co-morbid with self-injury, or may even predispose vulnerability for the behavioural pathology.

Clinical studies reveal that SIB is particularly prevalent in disorders where ongoing distress and pathological irritability are prominent features (Anderson \& Ernst 1994; Sovner \& Fogelman I996), and emotional stress may be a key trigger for SIB (Anderson \& Ernst 1994; Lindauer et al. I999). Furthermore, abnormal activity of the limbichypothalamic-pituitary-adrenal (LHPA) axis is a common characteristic of intellectually disabled self-injurers (Verhoeven et al. I999; Sandman et al. 2003, 2008; Symons et al. 2003; Kemp et al. 2008). However, it is not clear if LHPA axis dysregulation is a predisposing factor, or a consequence of SIB in these studies.

The relationship between emotional stress and self-injury is also seen in captive non-human primates. Self-injurious rhesus macaques display greater emotional responsiveness than their noninjurious counterparts do (Novak 2003), and the stress of relocation to novel housing produced longlasting increases in self-biting behaviour in these animals (Davenport et al. 2008). In addition, we recently reported that individual differences in innate stress responsiveness predict vulnerability for induction of self-injury in the pemoline model of SIB (Muehlmann et al. 20II). Despite these intriguing implications for the role of stress in aetiology and expression of SIB, remarkably little study has been conducted on the manner in which stress and self-injury interact in intellectually disabled populations.
We have conducted an extensive characterisation of the pemoline model in rats, and our studies reveal several features that converge with observations from intellectually disabled self-injurers. For example, each pemoline-treated rat targets a specific tissue site (e.g. one forepaw, but not the other), and repeatedly injures that site (Kies \& Devine 2004), in a manner that resembles the stereotypic body site preferences that are seen in human self-injurers (Symons \& Thompson I997). In addition, we found that pemoline-induced SIB is diminished by risperidone, valproate and topiramate (Muehlmann et al. 2008), drugs that are partially effective in human self-injurers (Ruedrich et al. 1999; McCracken et al. 2002; Shapira et al. 2002; Accardo 2003).

We now report two studies using the pemoline model of SIB in rats. In the first study, we used cytochrome oxidase (CO) histochemistry to identify brain regions that are impacted by chronic pemoline treatment in our model of SIB. The mitochondrial CO holoenzyme catalyses the final step in a process of electron transport that is tightly coupled to oxidative phosphorylation of adenosine diphosphate (yielding the highly energetic adenosine triphosphate) (Hatefi 1985). In brain tissue, this activity is required mostly for neuronal (as opposed to glial) activity, especially membrane repolarisation (WongRiley I989). In contrast to more transient markers of neuronal activity (e.g. c-fos), the overall level of $\mathrm{CO}$ provides a reliable index of sustained neuronal function. Therefore, it has been described as a useful marker of neuroplasticity (Konkle \& Bielajew 2004).

In the second study, we explored the impact of a prior history of stress exposure on the aetiology and expression of SIB, using the social defeat model. In this model, an experimentally naïve male rat (the intruder) is placed into the home cage of a larger male conspecific (the resident). The territorially dominant resident characteristically pins the intruder in a supine position (Miczek et al. 2004; Green \& Devine 2009; Marcinkiewcz et al. 2009). This social defeat procedure activates limbic, hypothalamic and brainstem structures that are implicated in processing of stressful stimuli in the intruder rat (Martinez et al. 2002), and it is thought to emulate social stressors that rats may be exposed to in their natural habitats (Huhman 2006). 
A. M. Muehlmann et al. - Stress and SIB in the pemoline model

\section{Methods}

Experiment I - cytochrome oxidase

Animals

Sixteen male Long-Evans (LE) rats weighing I50I75 g (Charles River Laboratories, Raleigh, NC, USA) were housed in a climate-controlled vivarium with a $\mathrm{I} 2 \mathrm{~h} / \mathrm{I} 2 \mathrm{~h}$ light/dark schedule (lights on at 6:00 h). Standard laboratory rat chow (Lab Diet 500I) and tap water were available ad libitum. The rats were pair-housed in standard polycarbonate cages $(43 \mathrm{~cm} \times 21.5 \mathrm{~cm} \times 25.5 \mathrm{~cm})$ during 6 days of acclimation to the housing facility. Starting on the first day of pemoline treatment, each rat was individually housed, in order to ascertain that any injuries were self-inflicted. All the experimental procedures were pre-approved by the Institutional Animal Care and Use Committee at the University of Florida, and all the procedures were conducted in accordance with the Guide for the Care and Use of Laboratory Animals (National Research Council 2OII).

\section{Drugs}

The indirect monoamine agonist pemoline (Fuller et al. 1978; Gilbert et al. 1978) (2-amino-5-phenylI,3-oxazol-4-one; Spectrum Chemicals, New Brunswick, NJ, USA) was suspended at a concentration of $50 \mathrm{mg} / \mathrm{ml}$ in warm peanut oil (held at approximately $36^{\circ} \mathrm{C}$ ), with constant stirring.

\section{Pemoline treatment and assays of self-injury}

On each of five consecutive mornings, approximately $2 \mathrm{~h}$ after the lights were turned on, each rat was examined for injuries, weighed and injected. Independent groups were treated with pemoline (200 mg/kg/day, s.c.; $n=\mathrm{IO}$ ) or vehicle (4 ml/kg/day, s.c.; $n=6)$. The dose of pemoline was selected on the basis of our previous studies (Kies \& Devine 2004), in which we found that reliable self-injury is produced by administration of $200 \mathrm{mg} / \mathrm{kg} / \mathrm{day}$, whereas a higher dose $(300 \mathrm{mg} / \mathrm{kg} /$ day $)$ produced very rapid onset of more severe self-injury, and a lower dose (IOO mg/kg/day) did not produce reliable SIB.

The injections were delivered to the nape of the neck or either flank on a rotating basis. The exami-
Table I Tissue injury rating scale (adapted from Kies \& Devine 2004)

\begin{tabular}{lll}
\hline Score & Severity & Description \\
\hline 0 & No injury & $\begin{array}{l}\text { No tissue damage } \\
\text { Slight oedema, pink moist skin, } \\
\text { involves small area } \\
\text { Mery mild injury }\end{array}$ \\
Mild injury & $\begin{array}{c}\text { Moderate oedema, slight } \\
\text { erythema, slightly denuded } \\
\text { skin, involves medium area } \\
\text { and/or involves multiple sites }\end{array}$ \\
& Moderate injury & $\begin{array}{c}\text { Substantially denuded skin, } \\
\text { substantial oedema and } \\
\text { erythema, large area and/or } \\
\text { involves multiple sites }\end{array}$ \\
& Severe injury & $\begin{array}{c}\text { Open lesion, requires immediate } \\
\text { euthanasia }\end{array}$ \\
& &
\end{tabular}

nations consisted of visual inspection of each rat's head, forepaws, ventrum, hindpaws and tail. Each rat was assigned a tissue damage score according to the presence and extent of injuries (see the rating scale in Table I). The placement of each selfinflicted injury and the number of sites of tissue damage were also recorded. In addition, the length of each injury was measured. Injuries on the ventrum were consistently oval, and injury on the tail (only one rat) encompassed the circumference of the tail along a portion of its length. Accordingly, the length of each injury at these sites provided an approximation of the relative sizes of injuries. Injuries on the paws were less regular in shape, so the length of the injury along the paw and up the limb was taken as an overall approximation of the extent of the injury.

The rats were checked again for injuries every evening, but these scores were not included in the data analysis (i.e. one score was counted per day from each rat). The evening scores (which resembled the morning scores quite closely) were used to make certain that no animal was allowed to severely injure itself overnight without intervention. In any case where an open lesion was identified (score $=4$ on the rating scale), the rat expressing the open lesion was immediately euthanised.

On the final morning of the experiment (day 6, 8:00 h-IO:00 h), each rat was checked again for injuries, and then rapidly decapitated. Each brain 
A. M. Muehlmann et al. - Stress and SIB in the pemoline model

was rapidly removed, frozen in 2-methylbutane at $-40^{\circ} \mathrm{C}$, and stored at $-80^{\circ} \mathrm{C}$.

\section{Cytochrome oxidase histochemistry}

Each brain was cryosectioned at $20 \mu \mathrm{m}$ in the coronal plane. Sections were mounted onto microscope slides on ice, and frozen at $-20^{\circ} \mathrm{C}$ overnight. The slides were then stained for CO histochemistry according to methods that were modified slightly from those of Gonzalez-Lima \& Jones (1994). Briefly, the frozen slides were fixed with $0.5 \%$ glutaraldehyde and I0\% sucrose in O.I $\mathrm{M} \mathrm{PO}_{4}$ buffer (5 min), rinsed with I०\% sucrose: O.I $\mathrm{M} \mathrm{PO}_{4}$ buffer ( $15 \mathrm{~min}$ ), and incubated with stirring for $60 \mathrm{~min}$ in the dark in pre-oxygenated O.I $\mathrm{M} \mathrm{PO}_{4}$ buffer containing $5 \%$ sucrose, $0.05 \%$ diaminobenzidine, $0.0075 \%$ cytochrome c, $0.002 \%$ catalase and $0.25 \%$ dimethyl sulfoxide. The slides were then fixed in I0\% buffered formalin with 10\% sucrose for $30 \mathrm{~min}$, dehydrated in graded $\mathrm{EtOH}(30,50,70,90$, $95,100 \%)$ and immersed in xylene (20 $\mathrm{min}$ ). The slides were coverslipped, and optical density (OD) was quantified for all regions of interest.

\section{Quantification of cytochrome oxidase}

Digital images of the sections were captured using a Northern Light R95 white trans-illuminator (Imaging Research Inc.) and a Photometrics CoolSnap CCD camera (Roper Scientific) connected to a personal computer. Semi-quantitative densitometry was performed using MCID Basic software (Imaging Research Inc.). Regions of interest were identified with reference to a standard rat brain atlas (Paxinos \& Watson 1998), and a standard sampling box was used in the data collection window for each of these regions. Bilateral OD measures were sampled from three sections per region. External background measures were taken from outside each section, used to correct each sample OD, and the measures from the six samples per region were averaged. During this procedure, the experimenter was unaware of the experimental conditions associated with any section.

\section{Data analyses}

Two of the pemoline-treated rats were euthanised on day 4 , because of injury. In these cases, the missing self-injury data were replaced by repeating the final score that was attained for each dependent measure through the end of the experiment. The brains were harvested and used for the CO assays, in the same manner as the brains of the rats that completed the experiment. This strategy was used to avoid the potential that the data would over- or underestimate the outcomes if the most severe self-injurers were eliminated. One additional rat was found dead on day 4. The cause was unknown, and it was removed from the experiment.

The percentage of rats that self-injured, mean tissue injury scores, numbers of injured sites and total length of injuries were plotted across days. Between-groups differences in tissue injury scores, number of injured sites and size of injuries were each evaluated using repeated-measures analyses of variance (RM-ANOvA). Effects were treated as statistically reliable when the $P$-values were less than 0.05. All significant effects were further analysed with Bonferroni post-tests.

The corrected OD measures were compared between groups using unpaired $t$-tests for each selected brain region. Between-groups differences were treated as statistically reliable when the $P$-values were less than 0.05 . In order to present the outcomes of all the 38 anatomical regions that were assayed, the averaged OD scores for the pemolinetreated rats were subtracted from the scores for the vehicle-treated rats, and the data were then graphed as difference scores.

\section{Experiment 2 - social defeat stress}

Animals

Twenty-three male LE rats weighing I50-I75 g (Charles River Laboratories) were pair-housed in the same manner as the rats in Experiment I, with a I $2 \mathrm{~h} / \mathrm{I} 2 \mathrm{~h}$ light/dark schedule (lights on at 6:00 h) and ad libitum food and water. These were the experimental rats and were used as the 'intruders'. An additional six vasectomised male LE rats weighing 400-450 g were pair-housed with six female LE rats weighing 200-225 g in a separate housing room with an opposite I $2 \mathrm{~h} / \mathrm{I} 2 \mathrm{~h}$ light/dark schedule (lights off at 6:00 h). These rats were used as the 'residents'. 
A. M. Muehlmann et al. - Stress and SIB in the pemoline model

\section{Social defeat}

Each male resident was trained to exhibit dominance behaviour by repeatedly removing the female and introducing a smaller male rat into the cage. All the residents used in this study consistently demonstrated dominance behaviour in pre tests of social dominance. Social defeat sessions with the experimental intruders were then run during the dark phase of the resident rats' light cycle, starting at approximately 7:00 h. At the beginning of each social defeat session, the female resident was removed from the home cage. Ten minutes later, an intruder rat was placed into the home cage with the resident rat. The rats were then allowed to interact for 5 min or until the intruder displayed a submissive behaviour three times. Submissive behaviour was defined as supine posture, with the resident male rat on top, for at least $2 \mathrm{~s}$. After this direct interaction phase, each intruder was removed, placed into a $10 \mathrm{~cm} \times 10 \mathrm{~cm} \times 15 \mathrm{~cm}$ double-layered wire mesh cage, and returned to the home cage of the resident male. This indirect interaction phase allowed the intruder rat to be out of physical contact but still experience stressful sensory stimuli. The intruder was maintained in the wire mesh cage until so min had elapsed from the start of the direct interaction phase, equalising the total duration of the stress session across rats. After the Io-min social defeat session concluded, both the female resident and the intruder were returned to their respective home cages. The intruder rats were subjected to social defeat once daily for 12 consecutive days, seeing each resident twice in that period, 6 days apart. Control rats were handled for 2 min each day for 12 days to ascertain that group differences could not be attributed to additional handling stress in the defeated rats.

\section{Drugs}

Pemoline was suspended at a concentration of $50 \mathrm{mg} / \mathrm{ml}$ in peanut oil. In order to get the pemoline into suspension, the solution was stirred overnight.

\section{Pemoline treatment and assays of self-injury}

Following the I2-day social defeat regimen, the rats were inspected, weighed and injected with pemoline at $150 \mathrm{mg} / \mathrm{kg}$ (s.c.) each morning for five consecutive days. In this experiment, a slightly lower dose was used than in the $\mathrm{CO}$ experiment, because the dose in that experiment $(200 \mathrm{mg} / \mathrm{kg} /$ day $)$ produced substantial SIB, and we anticipated that the stress exposure would exacerbate the expression of pemoline-induced SIB. These injections were administered at the nape of the neck and either flank on a rotating basis in the same manner as in Experiment I. The inspections were conducted in the same manner as in Experiment I, and like in Experiment I, they were repeated in the evening.

In this experiment, the inspections were videorecorded, still images of the injured tissue were captured, and the MCID Basic software was used to outline the injured tissue, and to calculate the area of injury in $\mathrm{mm}^{2}$. In addition, night-vision cameras were focused on the cages of the rats (one camera per cage), and 5-min time samples were recorded once every $3 \mathrm{~h}$ over the entire day. The duration of self-injurious oral contact and stereotypy were quantified during each videotaped interval by a trained observer. Self-injurious oral contact was defined as oral contact that stayed fixed on any specific site (e.g. forepaw) for longer than $2 \mathrm{~s}$. This was differentiated from grooming, which is oral contact that continues to move along a body part or from one body part to another. The stereotypy measure is a compilation of the duration of stereotyped bobbing and licking, wherein the rat either bobbed its head or licked the side or floor of the cage repeatedly, and the duration of stereotyped digging, sniffing, or burrowing through the bedding. The duration (in seconds) of self-injurious oral contact and stereotypy were each summed over the entire day (i.e. from the eight video samples) and divided by the total number of seconds recorded.

\section{Data analyses}

Six rats were euthanised before the end of the experiment (four stressed rats and two control rats) because of injury. In these cases, the missing data were replaced in the same manner as in Experiment I.

Between-groups differences in size of injuries, duration of self-injurious oral contact and duration of stereotypy were each evaluated using RMANOVA. Effects were treated as statistically reliable 
A. M. Muehlmann et al. - Stress and SIB in the pemoline model

when the $P$-values were less than 0.05 . All significant effects were further analysed with pre-planned Fisher's least significant difference post-tests.

\section{Results}

\section{Experiment I - cytochrome oxidase}

Tissue injury was observed by day 4 in all the pemoline-treated rats (Fig. IA). In the latter half of the experiment, the tissue injury scores $\left(F_{5,65}=22.48, P<0.000 \mathrm{I}\right)$, number of injured sites $\left(F_{5,65}=18.97, P<0.0001\right)$ and total size of injuries $\left(F_{5,65}=\mathrm{I} 5.63, P<0.000 \mathrm{I}\right)$ were greater than the corresponding measures in the control group (Fig. IB-D).

Cytochrome oxidase expression was significantly lower in the pemoline-treated rats in the caudate- putamen $(\mathrm{CPu})$, ventral pallidum (Fig. 2A), subregions of the septum and bed nucleus of stria terminalis (BNST) (Fig. 2B), specific hippocampal fields and hypothalamic nuclei (Fig. 2C), and the periaqueductal grey (PAG) (Fig. 2D). CO expression did not differ between the pemoline- and vehicle-treated groups in the substantia nigra, ventral tegmentum, core or shell of the nucleus accumbens septi (NAS), globus pallidus, limbic cortex, amygdaloid nuclei, subiculum, motor cortex, thalamic nuclei, or dorsal raphe (Fig. 2).

\section{Experiment 2 - social defeat stress}

All the pemoline-treated rats self-injured (Fig. 3A), and so the history of social defeat stress did not significantly affect the incidence of pemoline-
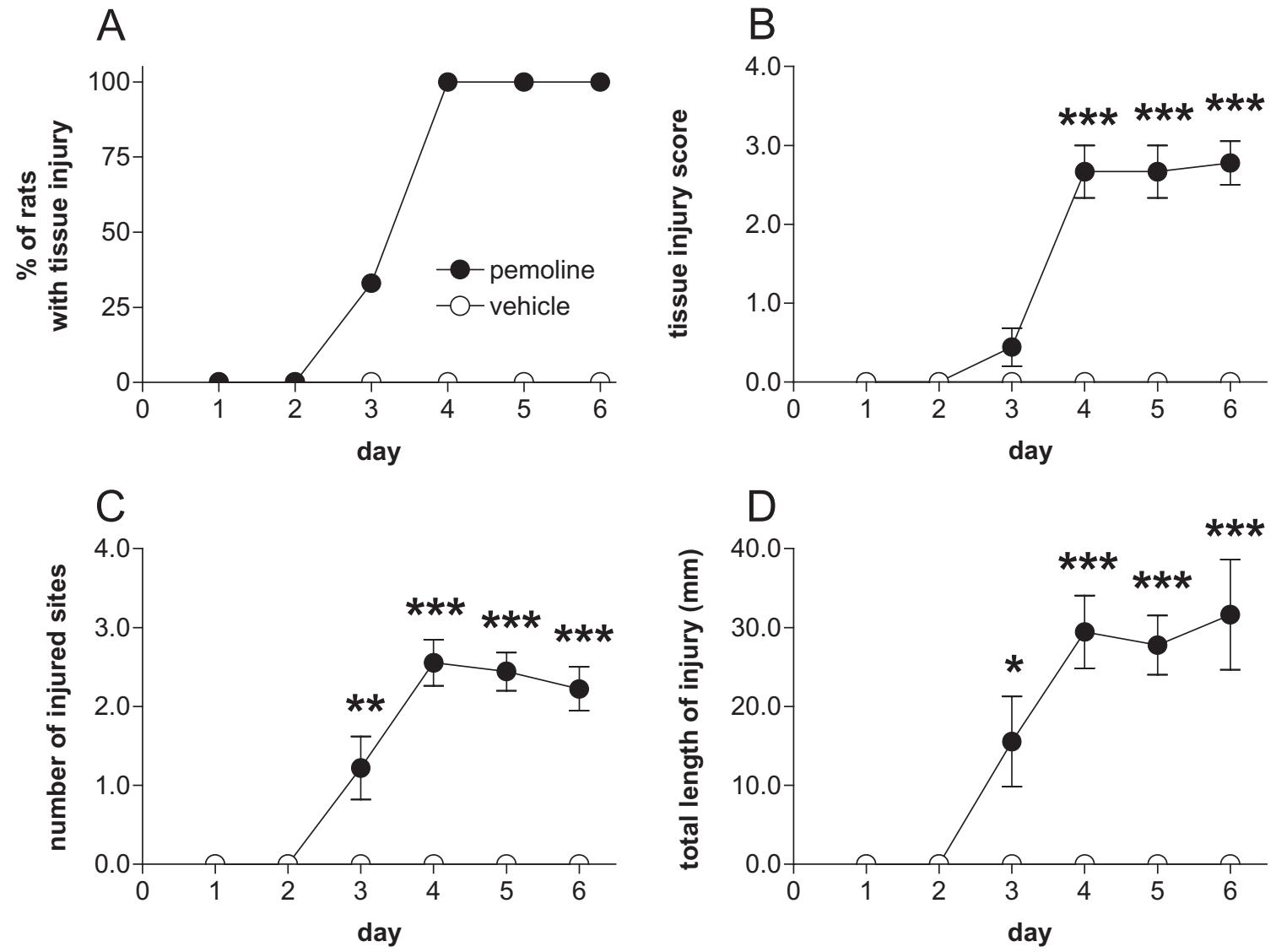

Figure I Incidence of pemoline-induced self-injury, tissue injury scores, number of injured sites and total length of injuries. Each measured increased across days. Self-injurious behaviour was observed in all the pemoline-treated rats, and in none of the vehicle-treated rats $(* P<0.05, * * P<0.01, * * * P<0.001)$.

(C) 20II The Authors. Journal of Intellectual Disability Research (C) 20I I Blackwell Publishing Ltd 
A. M. Muehlmann et al. - Stress and SIB in the pemoline model
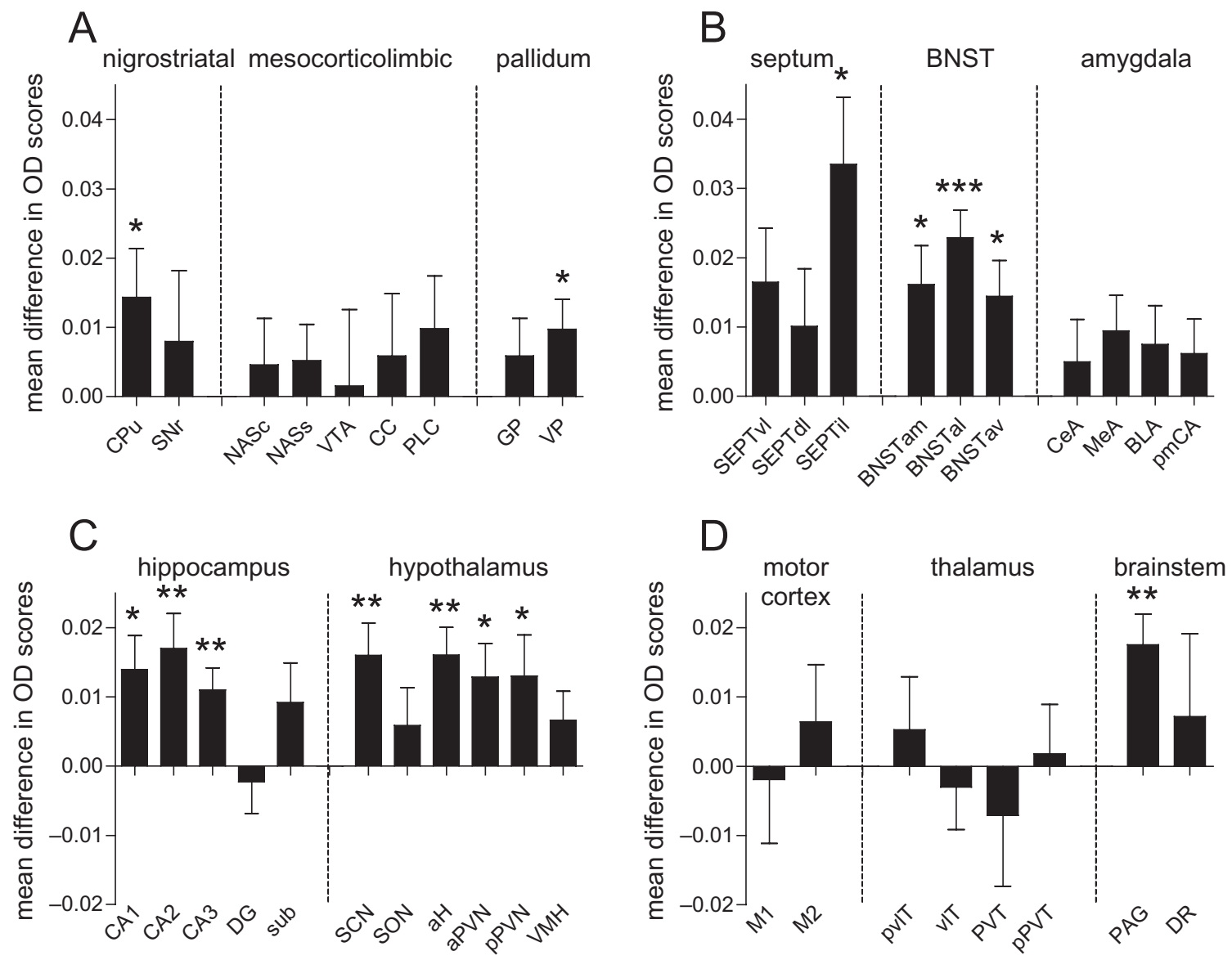

Figure 2 Cytochrome oxidase activity expressed as mean difference between the pemoline-treated and vehicle-treated rats. OD, optical density; $\mathrm{CPu}$, caudate-putamen; SNr, substantia nigra (pars reticulata); NASc, nucleus accumbens septi (core); NASs, nucleus accumbens septi (shell); VTA, ventral tegmental area; CC, cingulate cortex; PLC, prelimbic cortex; GP, globus pallidus; VP, ventral pallidum; SEPTvl, ventral lateral septum; SEPTdl, dorsal lateral septum; SEPTil, interior lateral septum; BNST, bed nucleus of stria terminalis; BNSTam, anteromedial BNST; BNSTal, anterolateral BNST; BNSTav, anteroventral BNST; CeA, central amygdala; MeA, medial amygdala; BLA, basolateral amygdala; pmCA, post-medial cortical amygdala; $\mathrm{CAI}-\mathrm{CA}_{3}$, cornu ammonis I-3; DG, dentate gyrus; sub, subiculum; SCN, suprachiasmatic nucleus; SON, supraoptic nucleus; aH, anterior hypothalamus; aPVN, anterior paraventricular nucleus; pPVN, posterior paraventricular nucleus; $\mathrm{VMH}$, ventromedial hypothalamus; $\mathrm{MI}$ and $\mathrm{M}_{2}, \mathrm{MI}_{\mathrm{I}}$ and $\mathrm{M}_{2}$ motor cortex; pvlT, posterior ventrolateral thalamus; vIT, ventrolateral thalamus; PVT, paraventricular thalamus; pPVT, posterior paraventricular thalamus; PAG, periaqueductal grey; DR, dorsal raphe. All values are expressed as group means $\pm \operatorname{SEM}(* P<0.05, * * P<0.01, * * * P<0.001)$.

induced SIB. However, the sizes of the injuries were significantly greater in the rats that had a history of social defeat stress (Fig. 3B). The RM-ANOvA revealed a significant main effect of time $\left(F_{10,210}=39.9\right.$ I,$\left.P<0.01\right)$ and a significant stress $\times$ time interaction $\left(F_{10,210}=2.095, P<0.05\right)$, wherein rats first displayed injured tissue around day 2 or 3 , and the size of injured tissue reached asymptote on day 4 or 5 . There were no significant differences in the amount of time spent in self- injurious oral contact between stressed and control rats (Fig. $\left.{ }_{3} \mathrm{C}\right)$. There was a significant main effect of time $\left(F_{4,84}=46.47, P<0.01\right)$ as all the rats began to show self-injurious oral contact on day 2 , which peaked on day 3, and continued throughout the experiment; however, there was no significant main effect of stress nor a stress $\times$ time interaction effect. There were also no differences in the duration of other pemoline-induced stereotypies (Fig. 3D). The RM-ANOVA revealed a significant main effect of time 
A. M. Muehlmann et al. - Stress and SIB in the pemoline model
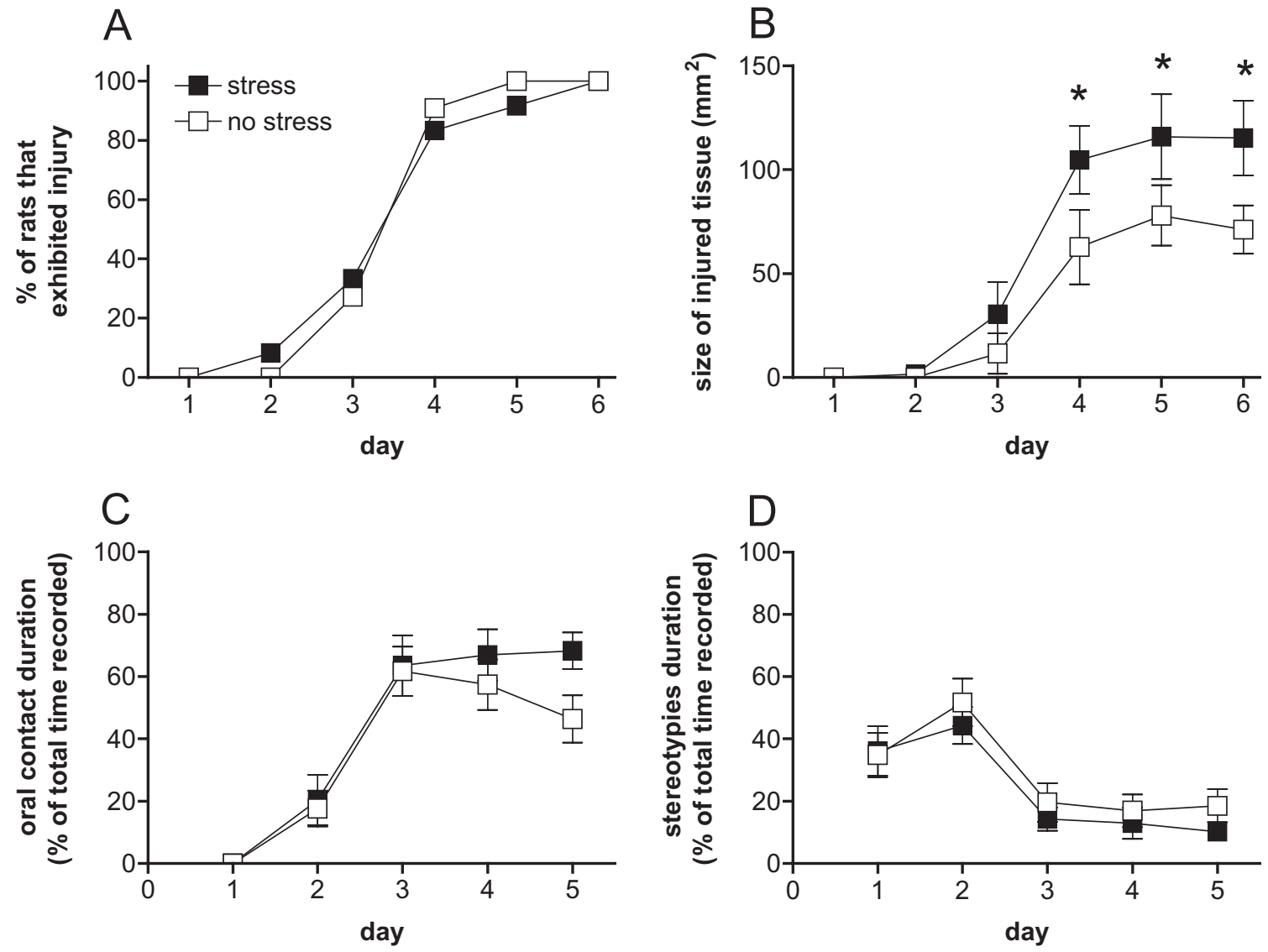

Figure 3 Effects of repeated social defeat stress on pemoline-induced self-injury and stereotypy. All the rats exhibited pemoline-induced self-injury (A); however, the rats with a history of repeated social defeat stress had larger areas of tissue damage (B). Repeated social defeat stress did not have any significant effect on pemoline-induced self-injurious oral contact (C) or whole body stereotypies (D). All values are expressed as group means \pm SEM $(* P<0.05)$.

$\left(F_{4,84}=\mathrm{I} 4.35, P<0.0 \mathrm{I}\right)$, as all the rats showed moderate amounts of stereotypy on days I and 2, which lessened on days $3-5$ as self-injurious oral contact duration increased. No significant main effect of stress or a stress $\times$ time interaction effect was found.

\section{Discussion}

In the CO histochemistry study, all the pemolinetreated rats exhibited self-injury, whereas none of the vehicle-treated rats injured (as expected). The anatomical assays revealed that sustained metabolic activity was suppressed in many of the 38 structures that were sampled from the self-injurious pemolinetreated rats, when compared with measures from the vehicle-treated rats. This suppression of neuronal activity was significant in one structure of the basal ganglia, and in a variety of limbic and limbicassociated structures. Although CO histochemistry provides only a gross characterisation of the regional effects of pemoline, it raises the possibility that direct or indirect impacts of the pemoline regimen in these regions may be associated with the induction of SIB.

Chronic pemoline treatment produced a statistically significant, but modest reduction in neuronal activity in the $\mathrm{CPu}$, and the effect was not significant in the NAS or frontal cortex. The paucity of effects in the striatum and frontal cortex may seem surprising, as pemoline is an indirect monoamine agonist (Fuller et al. 1978; Gilbert et al. 1978), and 
A. M. Muehlmann et al. - Stress and SIB in the pemoline model

these regions are targets of extensive dopaminergic innervation (Graybiel \& Ragsdale I979). Moreover, all measures of dopamine function were decreased in samples of $\mathrm{CPu}$ and NAS taken from individuals with Lesch-Nyhan syndrome (Lloyd et al. I98I; Ernst et al. 1996), and these biomarkers are generally ascribed a critical role in the aetiology of SIB in both Lesch-Nyhan syndrome (Schretlen et al. 2005) and animal models of the disorder (Breese et al. 1984). However, postsynaptic neurons expressing excitatory $\mathrm{D}_{1}$ and inhibitory $\mathrm{D}_{2}$ dopamine receptors are intermingled in the striatum and frontal cortex (Gaspar et al. 1995; Le Moine \& Bloch I995), so pemolinestimulated dopamine efflux would have opposing actions on these cells. As $\mathrm{CO}$ is most responsive to increases in membrane repolarisation (Wong-Riley I989), the opposing actions of dopamine on $\mathrm{D}_{1-}$ and $\mathrm{D}_{2}$-expressing cells could obscure the physiological effects of pemoline in these parts of the rat brain. CO histochemistry may be better suited to examine indirect impacts of pemoline on neurons that are downstream from these initial effects.

The other sites that exhibited significant suppression of neuronal metabolic activity were the ventral pallidum, interior lateral septum, BNST, subfields of the hippocampus, hypothalamic nuclei, and the PAG. On the other hand, other septal nuclei, dentate gyrus and subiculum, and the amygdala were not significantly impacted by chronic pemoline treatment. Thus, it seems that neuronal activity was suppressed only in a select subset of limbicassociated structures. Neuronal activity was not systematically altered in motor cortex or thalamus, suggesting a lack of involvement of these structures. Overall, the limbic/hypothalamic structures that exhibited significant pemoline-induced changes in neuronal metabolic activity, and the nigrostriatal and mesocorticolimbic systems, should each be examined more closely. The potential that these structures participate in the induction of SIB, and the specific biochemical alterations that occur in these structures require further analysis.

The suggestion that limbic structures may participate in pemoline-induced SIB is supported by evidence that emotional stress contributes to the expression of SIB in clinical populations (Favell et al. I982; Anderson \& Ernst 1994; Lindauer et al. 1999) and animal models (Stodgell et al. I998; Davenport et al. 2008). For example, escape from or avoidance of aversive situations (i.e. stressors) is recognised as a basic communicative function that drives SIB in intellectually disabled self-injurers (Favell et al. I982), and footshock stress increases the expression of SIB in the neonatal 6-hydroxydopamine (6-OHDA) lesion model in rats (Stodgell et al. I998). These observations led us to examine whether a background of emotional distress might influence the aetiology of SIB in the pemoline model. We selected the social defeat model of emotional stress because it activates the aforementioned limbic, hypothalamic and brainstem mechanisms (Martinez et al. 2002). Additionally, repeated social defeat increases basal plasma corticosterone concentrations (Covington \& Miczek 200I), and this is consistent with reports that LHPA axis function is dysregulated in intellectually disabled self-injurers (Verhoeven et al. 1999; Sandman et al. 2003, 2008; Symons et al. 2003; Kemp et al. 2008) [although it should be noted that both elevated and suppressed basal corticosterone concentrations have been found (Verhoeven et al. I999; Symons et al. 2003)].

In our study of the impact of social defeat on subsequent acquisition of pemoline-induced SIB, all the pemoline-treated rats self-injured, regardless of whether they were pre-exposed to social defeat stress or not. Therefore, it was not possible to see any stress-induced increase in the incidence of selfinjury. Likewise, there were no significant differences in the daily durations of self-injurious oral contact - perhaps also owing to a ceiling effect. However, the severity of tissue injury was significantly greater in the previously stressed rats, as indicated by the overall sizes of injured tissue in these rats. Thus, a history of psychosocial stress appears to contribute to the aetiology of SIB. When considered along with our recent finding that stresshyper-responsive rats are particularly vulnerable for pemoline-induced SIB, the results indicate that stress reduction may be an important intervention in populations that are at risk for development of SIB. These findings may also have implications for the role that impoverished institutional environments have played in the aetiology of SIB among the intellectually disabled.

Studies of the brain structures that are impacted by social defeat may also help to focus the search for neuroanatomical substrates that contribute to the aetiology of SIB. Interestingly, $c$-fos expression is 
A. M. Muehlmann et al. - Stress and SIB in the pemoline model

activated in many limbic, hypothalamic and brainstem structures after acute defeat. The response habituates in some of these structures (e.g. septum, lateral hypothalamus, central amygdala), but enduring effects are found in others (BNST, paraventricular nucleus, medial amygdala, PAG, raphe) (Martinez et al. 1998). As social defeat stress enhanced the severity of self-injury in rats that were subsequently treated with pemoline, the brain regions in which there is overlap between the persistent actions of social defeat, and the brain regions where pemoline produces reductions in sustained neuronal activity may be particularly important in mediating the contribution of stress to the aetiology of pemoline-induced SIB. These areas (BNST, paraventricular nucleus and PAG) may be particularly interesting targets for additional investigation.

\section{References}

Accardo P. (2003) Risperidone in children with autism and serious behavioral problems. Fournal of Pediatrics I42, 86-7.

Anderson L. T. \& Ernst M. (1994) Self-injury in LeschNyhan disease. Fournal of Autism and Developmental Disorders 24, 67-8I.

Breese G. R., Baumeister A. A., McCown T. J., Emerick S. G., Frye G. D. \& Mueller R. A. (I984) Neonatal-6hydroxydopamine treatment: model of susceptibility for self-mutilation in the Lesch-Nyhan syndrome. Pharmacology, Biochemistry, and Behavior 2I, 459-6I.

Covington H. E. III \& Miczek K. A. (200I) Repeated social-defeat stress, cocaine or morphine. Effects on behavioral sensitization and intravenous cocaine self-administration 'binges'. Psychopharmacology 158, 388-98.

Davenport M. D., Lutz C. K., Tiefenbacher S., Novak M. A. \& Meyer J. S. (2008) A rhesus monkey model of self-injury: effects of relocation stress on behavior and neuroendocrine function. Biological Psychiatry 63, 990-6.

Ernst M., Zametkin A. J., Matochik J. A., Pascualvaca D., Jons P. H., Hardy K. et al. (1996) Presynaptic dopaminergic deficits in Lesch-Nyhan disease. Nerw England Fournal of Medicine 334, I568-72.

Favell J. E., Azrin N. A., Baumeister A. A., Carr E. G., Dorsey M. F., Forehand R. et al. (1982) The treatment of self-injurious behavior. Behavior Therapy 13, 529-54.

Fuller R. W., Perry K. W., Bymaster F. P. \& Wong D. T. (1978) Comparative effects of pemoline, amfonelic acid and amphetamine on dopamine uptake and release in vitro and on brain 3,4-dihydroxyphenylacetic acid concentration in spiperone-treated rats. Fournal of Pharmacy and Pharmacology 30, 197-8.

Gaspar P., Bloch B. \& Le M. C. (1995) DI and D2 receptor gene expression in the rat frontal cortex: cellular localization in different classes of efferent neurons. European Fournal of Neuroscience 7, 1050-63.

Gilbert J. C., Allen J. M., Townsend B. G. \& Wyllie M. G. (1978) Drugs affecting the central nervous system: effects of pemoline and tricyclic antidepressants on nerve terminal adenosine triphosphatase activities and neurotransmitter release. Neuropharmacology 17, 4I9-2 I.

Gonzalez-Lima F. \& Jones D. (I994) Quantitative mapping of cytochrome oxidase activity in the central auditory system of the gerbil: a study with calibrated activity standards and metal-intensified histochemistry. Brain Research 660, 34-49.

Graybiel A. M. \& Ragsdale C. W. Jr (I979) Fiber connections of the basal ganglia. Progress in Brain Research 5I, 239-83.

Green M. K. \& Devine D. P. (2009) Nociceptin/Orphanin FQ and NOP receptor gene regulation after single or repeated social defeat exposure. Neuropeptides 43, 507I4.

Hatefi Y. (1985) The mitochondrial electron transport and oxidative phosphorylation system. Annual Review of Biochemistry 54, IOI5-69.

Huhman K. L. (2006) Social conflict models: can they inform us about human psychopathology? Hormones and Behavior 50, 640-6.

Kemp A. S., Fillmore P. T., Lenjavi M. R., Lyon M., Chicz-DeMet A., Touchette P. E. et al. (2008) Temporal patterns of self-injurious behavior correlate with stress hormone levels in the developmentally disabled. Psychiatry Research 157, I8I-9.

Kies S. D. \& Devine D. P. (2004) Self-injurious behaviour: a comparison of caffeine and pemoline models in rats. Pharmacology, Biochemistry, and Behavior 79, 58798.

Konkle A.T. \& Bielajew C. (2004) Tracing the neuroanatomical profiles of reward pathways with markers of neuronal activation. Reviews in the Neurosciences 15, 383-4I4.

Le Moine C. \& Bloch B. (I995) DI and D2 dopamine receptor gene expression in the rat striatum: sensitive cRNA probes demonstrate prominent segregation of DI and $\mathrm{D} 2$ mRNAs in distinct neuronal populations of the dorsal and ventral striatum. Fournal of Comparative $\mathrm{Neu}$ rology 355, 418-26.

Lesch M. \& Nyhan W. L. (I964) A familial disorder of uric acid metabolism and central nervous system function. American fournal of Medicine 36, 56I-70.

Lindauer S. E., DeLeon I. G. \& Fisher W. W. (1999) Decreasing signs of negative affect and correlated

(C) 20II The Authors. Journal of Intellectual Disability Research (C) 20I I Blackwell Publishing Ltd 
A. M. Muehlmann et al. - Stress and SIB in the pemoline model

self-injury in an individual with mental retardation and mood disturbances. Fournal of Applied Behavior Analysis 32, 103-6.

Lloyd K. G., Hornykiewicz O., Davidson L., Shannak K., Farley I., Goldstein M. et al. (I98I) Biochemical evidence of dysfunction of brain neurotransmitters in the Lesch-Nyhan syndrome. New England Fournal of Medicine 305, IIO6-II.

Marcinkiewcz C. A., Green M. K., Devine D. P., Duarte P., Vierck C. J. \& Yezierski R. P. (2009) Social defeat stress potentiates thermal sensitivity in operant models of pain processing. Brain Research I25I, II 2-2O.

Martinez M., Phillips P. J. \& Herbert J. (1998) Adaptation in patterns of c-fos expression in the brain associated with exposure to either single or repeated social stress in male rats. European fournal of Neuroscience 10, 20-33.

Martinez M., Calvo-Torrent A. \& Herbert J. (2002) Mapping brain response to social stress in rodents with c-fos expression: a review. Stress 5, 3-13.

Matson J. L., Baglio C. S., Smiroldo B. B., Hamilton M., Paclawskyj T. R., Williams D. et al. (I996) Characteristics of autism as assessed by the Diagnostic Assessment for the Severely Handicapped-II (DASH-II). Research in Developmental Disabilities 17, 135-43.

McCracken J. T., McGough J., Shah B., Cronin P., Hong D., Aman M. G. et al. (2002) Risperidone in children with autism and serious behavioral problems. New England Fournal of Medicine 347, 314-2I.

Miczek K. A., Covington H. E. III, Nikulina E. M. Jr \& Hammer R. P. (2004) Aggression and defeat: persistent effects on cocaine self-administration and gene expression in peptidergic and aminergic mesocorticolimbic circuits. Neuroscience and Biobehavioral Reviews 27, 787-802.

Muehlmann A. M., Brown B. D. \& Devine D. P. (2008) Pemoline-induced self-injurious behavior: a rodent model of pharmacotherapeutic efficacy. Fournal of Pharmacology and Experimental Therapeutics 324, 214-23.

Muehlmann A. M., Wilkinson J. A. \& Devine D. P. (20II) Individual differences in vulnerability for self-injurious behavior: studies using an animal model. Behavioural Brain Research 217, I48-54.

National Research Council (20II) Guide for the Care and Use of Laboratory Animals, National Academies Press, Washington, D.C.

Novak M. A. (2003) Self-injurious behavior in rhesus monkeys: new insights into its etiology, physiology, and treatment. American fournal of Primatology 59, 3-19.

Paxinos G. \& Watson C. (1998) The Rat Brain in Stereotaxic Coordinates, 4 th edn. Academic Press, San Diego, CA.

Rojahn J. \& Esbensen A. J. (2002) Epidemiology of selfinjurious behavior in mental retardation: a review. In: Self-Injurious Behavior: Gene-Brain-Behavior Relationships (eds S. R. Schroeder, M. L. Oster-Granite \& T. Thompson), pp. 4I-77. American Psychological Association, Washington, D.C.

Ruedrich S., Swales T. P., Fossaceca C., Toliver J. \& Rutkowski A. (1999) Effect of divalproex sodium on aggression and self-injurious behaviour in adults with intellectual disability: a retrospective review. Fournal of Intellectual Disability Research 43(Part 2), IO5-II.

Sandman C. A., Touchette P., Lenjavi M., Marion S. \& Chicz-DeMet A. (2003) Beta-endorphin and ACTH are dissociated after self-injury in adults with developmenta disabilities. American fournal on Mental Retardation 108, 4I4-24.

Sandman C. A., Touchette P. E., Marion S. D. \& ChiczDeMet A. (2008) The role of proopiomelanocortin (POMC) in sequentially dependent self-injurious behavior. Developmental Psychobiology 50, 680-9.

Schretlen D. J., Ward J., Meyer S. M., Yun J., Puig J. G., Nyhan W. L. et al. (2005) Behavioral aspects of LeschNyhan disease and its variants. Developmental Medicine and Child Neurology 47, 673-7.

Shapira N. A., Lessig M. C., Murphy T. K., Driscoll D. J. \& Goodman W. K. (2002) Topiramate attenuates selfinjurious behaviour in Prader-Willi syndrome. International fournal of Neuropsychopharmacology 5, I4I-5.

Sovner R. \& Fogelman S. (1996) Irritability and mental retardation. Seminars in Clinical Neuropsychiatry I, I05I4.

Stodgell C. J., Loupe P. S., Schroeder S. R. \& Tessel R. E. (I998) Cross-sensitization between footshock stress and apomorphine on self-injurious behavior and neostriatal catecholamines in a rat model of Lesch-Nyhan syndrome. Brain Research 783, I0-18.

Symons F. J. \& Thompson T. (1997) Self-injurious behaviour and body site preference. Fournal of Intellectual Disability Research 4I, 456-68.

Symons F. J., Butler M. G., Sanders M. D., Feurer I. D. \& Thompson T. (1999) Self-injurious behavior and Prader-Willi syndrome: behavioral forms and body locations. American fournal on Mental Retardation 104, 260-9.

Symons F. J., Sutton K. A., Walker C. \& Bodfish J. W. (2003) Altered diurnal pattern of salivary substance $P$ in adults with developmental disabilities and chronic selfinjury. American fournal on Mental Retardation 108, I3-18.

Verhoeven W. M. A., Tuinier S., van den Berg Y.W. M. M., Coppus A. M.W., Fekkes D., Pepplinkhuizen L. et al. (1999) Stress and self-injurious behavior; hormonal and serotonergic parameters in mentally retarded subjects. Pharmacopsychiatry 32, 13-20.

Wong-Riley M. T. (1989) Cytochrome oxidase: an endogenous metabolic marker for neuronal activity. Trends in Neurosciences I2, 94-I0I.

Accepted I6 August 2011

(C) 20II The Authors. Journal of Intellectual Disability Research (C) 20I I Blackwell Publishing Ltd 